\title{
有机盐对水/AOT/醇反相微乳体系电导行为的影响
}

\author{
张晓光董金风* 张高勇周晓海洪昕林 \\ (武汉大学化学与分子科学学院, 武汉 430072)
}

\begin{abstract}
摘要 用二(2-乙基已基)琥珀酸酯磺酸钠(AOT)为表面活性剂研究了以正构醇(己醇、庚醇、辛醇、癸醇)为连续相 的微乳体系的电导行为, 结果表明只有水/AOT/癸醇体系有水诱导的电导渗滤现象. 研究了有机盐(胆酸钠、水杨 酸钠)及温度对电导行为的影响, 发现庚醇、辛醇体系电导率随胆酸钠浓度的增加而减小, 而癸醇体系电导率不 受影响; 庚醇、癸醇体系的电导率随水杨酸钠浓度的增加而增大; 在 $5 \sim 40{ }^{\circ} \mathrm{C}$ 范围内 $\ln \sigma$ (电导率的自然对数)与温 度成很好的线性关系, 无论有机盐存在与否都没有温度诱导的渗滤现象. 根据 Arrhenius-type 公式估算了体系的 电导活化能.
\end{abstract}

关键词: 醇, 微乳液, 电导率, 渗滤, 有机盐, AOT

中图分类号: 0648

\section{The Effect of Organic Salts on the Conductance of the Water/A0T/ Alkanol W/O Microemulsion}

\author{
ZHANG, Xiao-Guang DONG, Jin-Feng * ZHANG, Gao-Yong ZHOU, Xiao-Hai HONG, Xin-Lin \\ (College of Chemistry and Molecular Sciences, Wuhan University, Wuhan 430072, P. R. China)
}

\begin{abstract}
The conductivity behaviour of water-in-alkanol (hexanol, heptanol, octanol, decanol) microemulsions, in which AOT was employed as surfactant, was investigated. The percolation phenomenon induced by water was found only in the water/AOT/decanol system. The effects of organic salts (sodium cholate and sodium salicylate) and temperature on conductance were then studied. In heptanol and octanol systems the conductivity was decreased with the increase of sodium cholate concentration, whereas it was increased in the case of sodium salicylate. In decanol system the conductivity was hardly changed in the presence of both organic salts. The values of $\ln \sigma$ had a linear correlation with temperature in the range of $5 \sim 40{ }^{\circ} \mathrm{C}$. No percolation threshold induced by temperature was detected either in the absence or in the presence of organic salts. The activation energy for conductance was estimated according to the Arrhenius-type equation.
\end{abstract}

Keywords: Alkanol, Microemulsion, Conductivity, Percolation, Organic salts, AOT

反相微乳液在药物、化妆品、干洗、三次采油、胶 束催化和酶催化等领域的广泛应用引起了人们的关 注. 目前的研究大多集中在以二(2-乙基已基)琥珀酸 酯磺酸钠 (AOT) 为表面活性剂、中长链烷烃为连续 相的反相微乳液体系, 对于表面活性剂在烷烃中的 物理化学性质如增溶行为 ${ }^{[1-2]}$ 和电导行为 ${ }^{[3-5]}$ 已经有系
统的研究报道. 中、长链的醇常被用作助表面活性剂 来调节界面膜的性质. 而在实际应用中, 醇往往被直 接用来作为溶剂或反应介质, 表面活性剂在醇中的 行为则较少见报道[ ${ }^{[68]}$. 电导渗滤现象是反相微乳液的 一个重要的物理化学性质, 它是指在一定温度下, 分 散相(水)体积分数达到阀值体积时或者一定组成下

Received: May 13, 2005; Revised: July 6, 2005. $\quad$ *Correspondent, E-mail: Jfdong@whu. edu. cn; Tel: 027-87218534.

科技部“十五”攻关计划滚动项目(2004BA308A25-14)资助 
温度达到阀值温度时体系电导率会突然增大 100 1000 倍的现象 ${ }^{[0-10]}$. 关于烷烃体系的电导渗滤研究已 有大量报道 ${ }^{[11-12]}$. 人们通常认为发生电导渗滤时, 含 有分散相(水)液滴形成簇或聚集体, 离子由一个液 滴弹跳到另一个液滴 ${ }^{[13-15]}$ 或者在液滴“融合-分裂”过 程 ${ }^{[3]}$ 中离子进行质量转移. Jada 等 ${ }^{[3,16}$ 通过时间分辨苂 光技术测定了液滴间物质交换速率常数 $\left(k_{\mathrm{e}}\right)$, 表明其 值大于 $1 \times 10^{9} \sim 2 \times 10^{9} \mathrm{~L} \cdot \mathrm{mol}^{-1} \cdot \mathrm{s}^{-1}$ 时体系才可能发生渗 滤. Kataoka 等 ${ }^{[17]}$ 通过 PGSE-NMR 技术测定了 $\mathrm{D}_{2} \mathrm{O} /$ $\mathrm{AOT} /$ 癸烷体系中水的自扩散, 表明体系电导率的增 加主要是水的自扩散, 即主要的带电载体是 $\mathrm{Na}^{+}$. 这 些研究都证实了发生渗滤时电导率的突然增加主要 是反离子在液滴 “融合-分裂”过程中进行质量转移. 在最近 20 年里, 添加剂对烷烃体系渗滤影响的研究 较多 ${ }^{[18-21]}$, 结果表明添加剂如化合物 bile 盐(胆酸钠 和脱氧胆酸钠) 通过降低水的渗滤阀值体积或阀值 温度而促进渗滤, 而芳香族化合物如甲苯、二甲苯、 苯、荎、水杨酸钠等通过增加水的渗滤阀值体积或阀 值温度而延迟或阻止渗滤. 关于添加剂对电导渗滤 的影响, 文献[15, 19-20, 22]提出了相关解释机理, 认 为bile盐在液滴融合过程中形成了 “通道”, 有助于离 子的传递, 而芳香族化合物通过 “阻断”液滴的融合 而延迟了离子的传递.

Ray 等响研究了水/AOT/醇体系的电导行为, 结 果表明戊醇和已醇体系没有水诱导的渗滤现象, 庚 醇、辛醇和癸醇体系有水诱导的渗滤. 关于体系发生 渗滤的机理作者并没有给出具体的解释. 胆酸钠和 水杨酸钠对烷烃体系电导渗滤有截然相反的作用, 但对水/AOT/醇体系的影响还不清楚. 本文研究了 水/AOT/醇体系在有无有机盐胆酸钠 $(\mathrm{NaC})$ 和水杨 酸钠 $(\mathrm{NaSal})$ 存在时的电导渗滤, 对体系发生渗滤的 机理以及有机盐的影响进行了初步的讨论; 同时研 究了温度对水/AOT/醇体系电导行为的影响, 根据 Arrhenius-type 公式估算了体系的电导活化能.

\section{1 实验部分}

\section{1 试剂和仪器}

表面活性剂 AOT, 纯度 99\%(w), 美国 Sigma 公 司, 真空干燥箱中 $60{ }^{\circ} \mathrm{C}$ 下干燥 $48 \mathrm{~h}$, 咜存在放有 $\mathrm{P}_{2} \mathrm{O}_{5}$ 的真空干燥器中, 使用前未作进一步处理. 已 醇、庚醇、辛醇及癸醇, 中国医药集团上海化学试剂 公司进口分装, 使用前经过蒸馏后用 $\mathrm{CaO}$ 干燥; 胆 酸钠, 纯度 $99 \%(w)$, Acros Organics 产品; 水杨酸钠,
分析纯, 中国医药集团上海化学试剂公司. 所有溶液 用二次蒸馏水(其电导率值 $30{ }^{\circ} \mathrm{C}$ 时为 $2 \sim 4 \mu \mathrm{S} \cdot \mathrm{cm}^{-1}$ ) 配制.

DDS-6700 数字电导率仪, 上海雷磁新泾仪器 有限公司; 流变仪 RS600, 美国 Thermo Electron Corporation; 动态光散射仪 NANO ZS, 英国 Malvern Instruments, He-Ne 激光器, 波长为 $633 \mathrm{~nm}$, 测量角 度为 $173^{\circ}$.

\section{2 测定方法}

为使研究对象在反相微乳液区, 根据文献 [7, 23 ] 选取了 $\mathrm{AOT}$ 在醇相中的浓度为 $0.2 \mathrm{~mol} \cdot \mathrm{L}^{-1}$. 含有 $0.2 \mathrm{~mol} \cdot \mathrm{L}^{-1} \mathrm{AOT}$ 的醇溶液在室温配制. 利用数字电 导率仪测定微乳液的电导率 $(\sigma)$, 测定时向一定量的 AOT 醇溶液中用微量进样器逐渐加人水(有机盐溶 液), 保持温度为 $(30.0 \pm 0.1){ }^{\circ} \mathrm{C}$, 加人的水与 $\mathrm{AOT}$ 的 摩尔比为 $x$. 测定不同温度下电导率时, 温度范围为 $5.0 \sim 40.0{ }^{\circ} \mathrm{C}$, 控温精度为 $\pm 0.1{ }^{\circ} \mathrm{C}$, 在磁力搅拌下进行. 研究水及有机盐对单纯醇体系电导率影响时, 取一 定量的醇, 每次用微量进样器向醇中加人一定体积 的水 (有机盐溶液), 加人的水与醇的摩尔比为 $x^{\prime}$.

利用流变仪通过同轴圆筒法测定醇的黏度, 实 验温度为 $(30.0 \pm 0.1){ }^{\circ} \mathrm{C}$. 微乳液粒径测试时, 测试前样 品用 $0.20 \mu \mathrm{m}$ 膜过滤除尘, 测定扩散系数 $(D)$, 然后根 据Stokes-Einstein 公式获得流体力学直径 $(d)$, 测试温 度为 $(30.0 \pm 0.1){ }^{\circ} \mathrm{C}$.

\section{2 结果与讨论}

\section{1 水含量诱导的 W/O 微乳液电导渗滤}

图 1 是水/AOT/醇体系的 $\ln \sigma$ 随 $x$ 的变化图; 为 了说明水对单纯醇体系电导率的影响, 图 2 示出了

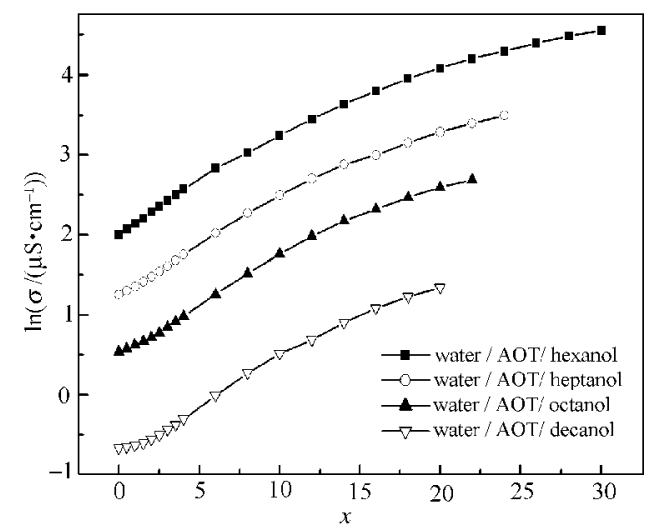

图 1 水/AOT/醇体系的 $\ln \sigma$ 随 $x$ 的变化

Fig.1 Dependence of $\ln \sigma$ on $x$ in water/AOT/alkanol systems

$x$ is the molar ratio of water to AOT. 


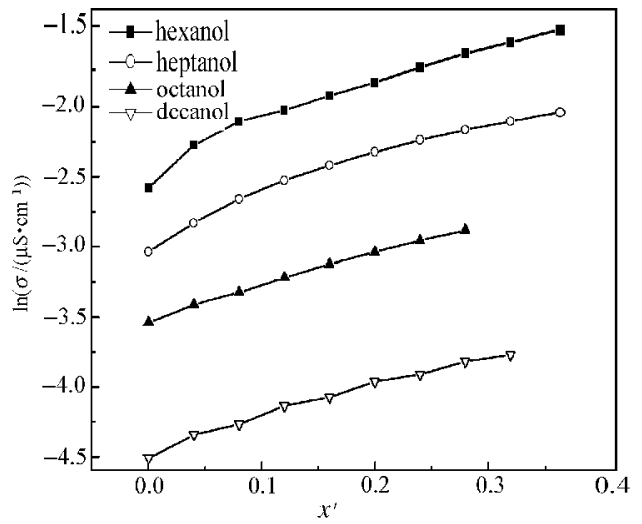

图 2 单纯的醇体系 $\ln \sigma$ 随 $\boldsymbol{x}^{\prime}$ 的变化

Fig.2 Dependence of $\ln \sigma$ on $x^{\prime}$ in pure alkanol systems

$x^{\prime}$ is the molar ratio of water to alkanol.

没有 $\mathrm{AOT}$ 时单纯醇体系 $\ln \sigma$ 随 $x^{\prime}$ 的变化. 对于单纯 醇体系, $\ln \sigma$ 几乎都随 $x^{\prime}$ 的增加而直线上升, 其中己 醇的 $\ln \sigma$ 值最大, 癸醇的 $\ln \sigma$ 值最小, 表明中等链长 的醇其导电能力随醇碳原子数的增加而降低. 对比 图 1 和图 2 可以看出, 单纯醇体系电导率远远低于 含有 AOT 的电导率, 表明 AOT 作为一种电解质极 大地提高了体系的电导率, 对于水/AOT/醇体系来 说醇对体系电导率的贡献很小, 主要是 AOT 对电 导率的贡献. 从 4 种醇的介电常数及黏度数据(见表 1)可以看出, 随着醇碳原子数的增加, 介电常数逐渐 变小, 黏度逐渐增大, 表明醇的极性和亲水性逐渐降 低, 亲油性增加. 结合图 2 和表 1 可知, 对于离子导 电来说, 己醇、庚醇和辛醇是比较好的介质, 容易促 进离子导电的发生. 癸醇与其它 3 种醇相比极性最 小, 水在其中溶解度最小, 亲油性较强, 不利于离子 导电.

对于水/AOT/癸醇体系, 当 $x<2$ 时, 随 $x$ 增加 $\ln \sigma$ 基本不变; 当 $x$ 进一步增加时, $\ln \sigma$ 随之单调增 加, 体系有明显的电导渗滤现象. 已醇、庚醇及辛醇 体系中的 $\ln \sigma$ 随 $x$ 增加而单调增加, 没有观察到渗 滤现象. Ekwall等 ${ }^{[23]}$ 认为在水/AOT/癸醇体系中, 醇 的羟基基团和 AOT 的极性头相互作用会使原有的

\section{表 1 醇的物理性质数据}

Table 1 The physical properties of alkanols

\begin{tabular}{cccc}
\hline Alkanol & $\begin{array}{c}\text { Dielectric } \\
\text { constant }\left(20{ }^{\circ} \mathrm{C}\right)\end{array}$ & $\eta^{\Phi} /(\mathrm{mPa} \cdot \mathrm{s})$ & $\begin{array}{c}\text { Molecular } \\
\text { volume }\left(\mathrm{nm}^{3}\right)\end{array}$ \\
\hline hexanol & 13.03 & 4.08 & 0.2078 \\
heptanol & 11.75 & 5.15 & 0.2354 \\
octanol & 10.30 & 6.56 & 0.2611 \\
decanol & 8.10 & 9.81 & 0.3156 \\
\hline
\end{tabular}

AOT 胶束分裂成小的胶束. 对于我们所研究的水/ AOT/醇体系, 醇既作为油相, 又作为助表面活性剂 参与胶束形成. 由于己醇、庚醇和辛醇的极性较强, 醇分子较容易渗透进人胶束单分子层, 羟基和 AOT 的极性头磺酸基有强烈的相互作用导致比较小的液 滴(胶束)形成, 小的液滴与醇相极性相近. 又由于液 滴间吸引力的作用, 这种小的液滴随水含量的增加 很容易相互碰撞融合在一起形成有利于离子迁移的 通道 ${ }^{[7]}$, 电导率快速增加, 因此没有观察到渗滤发生. 与其它 3 种醇相比, 癸醇极性较低, 碳链较长, 分子体 积较大(根据醇的摩尔分子量和其密度估算了醇的分 子体积, 见表1), 由于空间位阻的影响使得醇分子渗 透进人胶束的界面层变得困难, 导致它与 AOT 分 子的磺酸基相互作用比较弱, 形成的液滴与癸醇相 极性差异较大, 液滴间吸引力较小, 液滴碰撞融合在 一起形成通道变得困难, 电导率较低, 故在癸醇体系 中观察到了渗滤发生. 另外液滴的聚集行为与液滴 的扩散速率有关, 而扩散系数与体系的黏度成反比, Stokes-Einstein 公式给出了扩散系数与黏度的关系:

$$
D=\frac{k T}{3 \pi \eta d}
$$

其中, $D$ 是扩散系数, $k$ 是玻尔兹曼常数, $T$ 是绝对温 度, $\eta$ 是连续相(醇)的黏度, $d$ 是液滴流体动力学直 径. 当 $x=0$ 时液滴流体动力学直径及扩散系数结果 见表 2, 已醇的液滴直径最小, 庚醇、辛醇、癸醇体系 液滴直径基本一致, 扩散系数随碳链的增加而减小. 结合表 1 黏度数据和式(1)可知, 4 种醇体系在相同 温度下, 当体系的液滴直径改变不大时, 黏度最大的 癸醇体系具有最小的液滴扩散系数, 液滴相互吸引 聚集的运动最慢, 导致液滴相互碰撞形成离子转移 的通道变得困难, 因此在癸醇体系观察到渗滤现象.

\section{2 有机盐( $\mathrm{NaC}$ 和 $\mathrm{NaSal}$ )对水/AOT/醇电导行 为的影响}

胆酸钠和水杨酸钠的结构见图 3. 二者都含有 差基和羧基, 但是 $\mathrm{NaC}$ 分子比较大, 含有环状结构

\section{表 2 水/AOT/醇体系粒径 $(d)$ 和扩散系数 $(D)(x=0)$}

Table 2 The hydrodynamic diameter $(d)$ and the diffusion coefficient $(D)$ of water/AOT/alkanols systems

\begin{tabular}{ccc}
\hline water/AOT/alkanol & $d / \mathrm{nm}$ & $10^{-6} \mathrm{D} /\left(\mathrm{nm}^{2} \cdot \mathrm{s}^{-1}\right)$ \\
\hline water/AOT/hexanol & 3.5 & 30.4 \\
water /AOT/ heptanol & 3.8 & 22.5 \\
water /AOT/ octanol & 3.9 & 17.1 \\
water/AOT/decanol & 3.9 & 11.5 \\
\hline
\end{tabular}




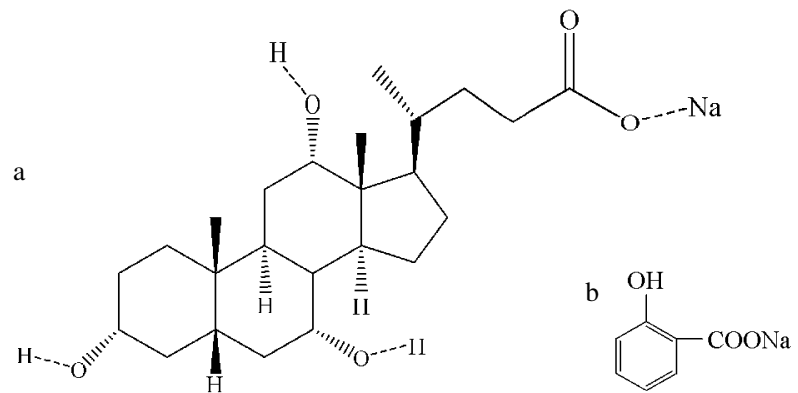

图 3 胆酸钠 $(a)$ 和水杨酸钠 $(b)$ 的结构

Fig.3 The structure of sodium cholate (a)and sodium salicylate (b)

以及三个羟基. 前面提到在烷烃体系中, $\mathrm{NaC}$ 促进 渗滤而 NaSal 抑制渗滤的发生, 这是因为 $\mathrm{NaSal}$ 分 子中含有一个羟基具有 “阻断” 液滴融合作用, 而 $\mathrm{NaC}$ 含有三个羟基具有促进渗滤作用 ${ }^{[18-21]}$.

2.2.1 胆酸钠对水 $/ A O T /$ 醇体系及单纯醇体系的电导行为
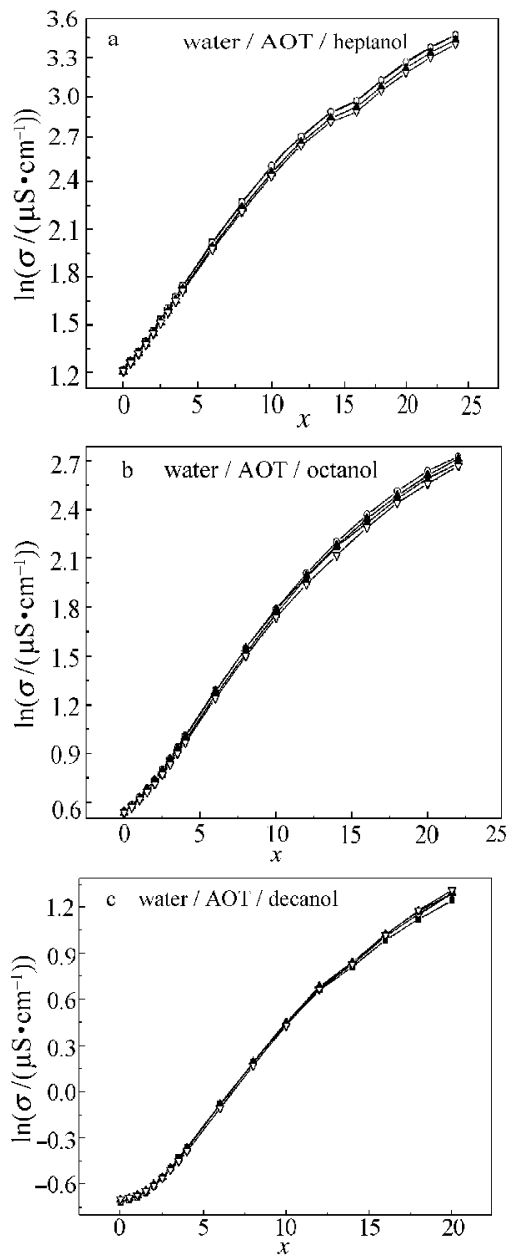

图 4 胆酸钠存在时水/AOT/醇体系 $\ln \sigma$ 随 $x$ 的变化

Fig.4 Dependence of $\ln \sigma$ on $x$ in water/AOT/ alkanols in the presence of sodium cholate $(\mathrm{NaC})$ $c(\mathrm{NaC}) /\left(\mathrm{mol} \cdot \mathrm{L}^{-1}\right)$

$\mathbf{\square}) 0 ; \bigcirc) 0.01 ; \mathbf{\Delta}) 0.1 ; \nabla) 0.2$
的影响

不同浓度 $\mathrm{NaC}$ 对水/AOT/醇体系电导行为的影 响见图 4; 为了说明庚醇、辛醇、癸醇与 $\mathrm{NaC}$ 间是否 存在相互作用, 图5给出了 $\mathrm{NaC}$ 对单纯醇体系电导行 为的影响. 对于水/AOT/庚醇(辛醇)体系, $\ln \sigma$ 随 $\mathrm{NaC}$ 浓度增加而逐渐下降, 而水/AOT/癸醇体系 $\ln \sigma$ 基本 不随 $\mathrm{NaC}$ 浓度的改变而改变. 对于单纯的庚醇和辛 醇体系(图 $5 \mathrm{a}, \mathrm{b}$ ), 当 $\mathrm{NaC}$ 浓度由0逐渐增加到 $0.2 \mathrm{~mol}$ ・ $\mathrm{L}^{-1}$ 时, $\ln \sigma$ 大幅增加; 而对于癸醇体系(图 5c), 0.001 $\mathrm{mol} \cdot \mathrm{L}^{-1} \mathrm{NaC}$ 对电导行为基本没有影响, $\mathrm{NaC}$ 浓度 高于 $0.001 \mathrm{~mol} \cdot \mathrm{L}^{-1}$ 时 $\ln \sigma$ 才迅速增加. 由此推测单 纯的醇体系 $\ln \sigma$ 增加主要是胆酸钠的贡献, 即 $\mathrm{Na}^{+}$ 的贡献.

由图3 (a) 可以看出 $\mathrm{NaC}$ 分子比较大, 含有环状结 构以及三个羟基和一个羧基. $\mathrm{NaC}$ 分子可能吸附在 表面活性剂界面膜表面, 差基与 AOT 的极性头磺
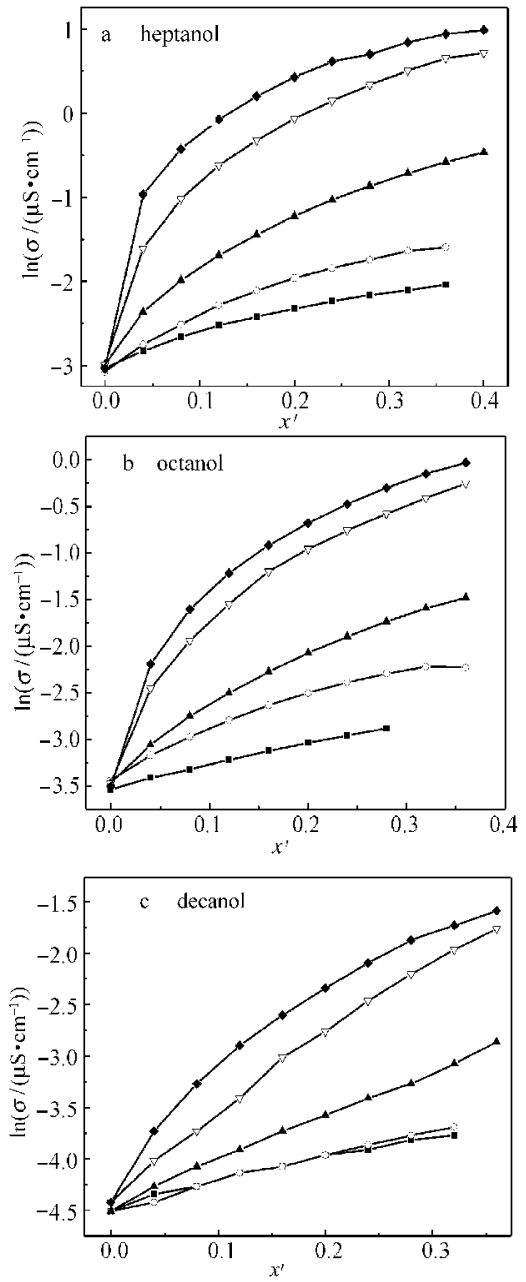

图 5 胆酸钠存在时单纯醇体系 $\ln \sigma$ 随 $\boldsymbol{x}^{\prime}$ 的变化

Fig.5 Dependence of $\ln \sigma$ on $x^{\prime}$ in pure alkanols systems in the presence of $\mathrm{NaC}$

$\left.\left.\left.\left.c(\mathrm{NaC}) /\left(\mathrm{mol} \cdot \mathrm{L}^{-1}\right): \mathbf{\square}\right) 0 ; \bigcirc\right) 0.001 ; \mathbf{\Lambda}\right) 0.01 ; \nabla\right) 0.1$ 

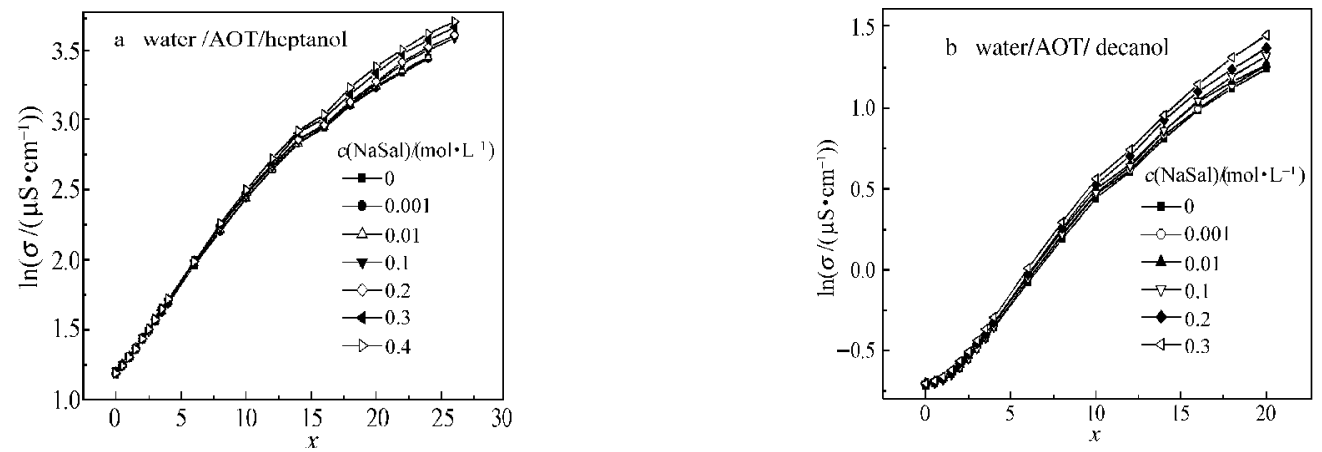

图 6 水杨酸钠存在时水/AOT/醇体系 $\ln \sigma$ 随 $x$ 的变化

Fig.6 Dependence of $\ln \sigma$ on $x$ in water/AOT/ alkanols in the presence of NaSal

酸基相互作用可能位于栅栏层. 由于庚醇和辛醇极 性比较强, 与 AOT 的极性头磺酸基有强烈的相互 作用使表面活性剂界面膜比较致密; $\mathrm{NaC}$ 分子比较 大, 加人后使界面排列得更加紧密, 不利于 $\mathrm{Na}^{+}$在液 滴碰撞融合过程中进行迁移. 另外 $\mathrm{NaC}$ 分子中的差 基和水形成氢健, 减少了 AOT 分子与水的结合, 使 得 AOT 分子的解离度降低, 液滴中 $\mathrm{Na}^{+}$数目降低, 导致电导率降低. 而癸醇极性相对较小, 与 AOT 极 性头相互作用形成的界面膜可能比较疏松, 加人 $\mathrm{NaC}$ 后虽使界面排列紧密, 但对 $\mathrm{Na}^{+}$迁移影响不大.

\subsubsection{NaSal 对水/AOT/庚醇(癸醇)体系电导行为的影响}

图 6 为不同浓度水杨酸钠对水/AOT/庚醇和水/ $\mathrm{AOT} /$ 癸醇体系电导行为的影响. 对于水/AOT/庚醇 和水/AOT/癸醇体系, 当 $\mathrm{NaSal}$ 浓度低于 $0.01 \mathrm{~mol}$ $\mathrm{L}^{-1}$ 时, $\ln \sigma$ 基本不受 $\mathrm{NaSal}$ 浓度的影响; 当 $\mathrm{NaSal}$ 浓 度高于 $0.1 \mathrm{~mol} \cdot \mathrm{L}^{-1}$ 时, $\ln \sigma$ 随之增加. 另外 $\mathrm{NaSal}$ 对 水/AOT/癸醇体系电导渗滤没有影响. 一方面 $\mathrm{NaSal}$ 为水溶性的添加剂, 加人后破坏了液滴内水的结构, 降低了AOT的亲油性, 使液滴间作用力降低 ${ }^{[24]}$, 同时 增加了胶束界面膜的柔性, 为 $\mathrm{Na}^{+}$提供了自由运动 的环境; 另一方面 $\mathrm{NaSal}$ 增加了液滴中的 $\mathrm{Na}^{+}$浓度, $\mathrm{Na}^{+}$溶解在水中同水一起迁移, 是主要的带电载体,

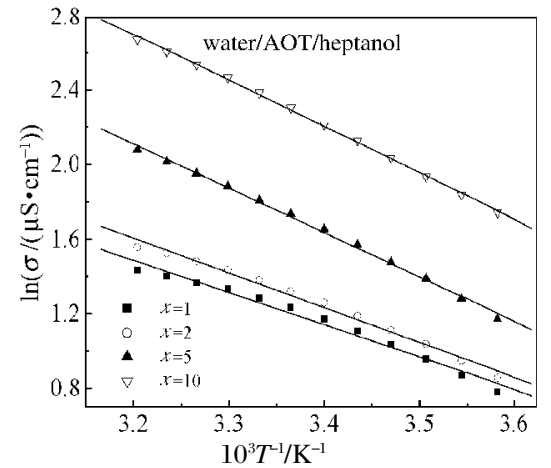

在导电过程中起主要作用 ${ }^{[17]}$, 随 $\mathrm{NaSal}$ 浓度的增加, 体系电导率增加.

\section{3 温度对水/AOT/庚醇(癸醇)体系电导行为的影响}

在渗滤过程中微乳液液滴间的相互作用可以用 “瞬时融合-质量转移-分裂”过程来描述, 其间 $\mathrm{Na}^{+}$穿 过界面膜进行迁移. 与此过程相关的活化能指的是 $\mathrm{Na}^{+}$迁移所需的能量以及液滴经过融合后再次分裂 所需能量之和 ${ }^{[25]}$. 发生渗滤时水分子自扩散的突然增 加导致了 $\mathrm{Na}^{+}$自扩散的增加. 因此在温度 $T$ 时, 迁移 率 $(\mu)$ 、扩散系数 $(D)$ 和电导率 $(\sigma)$ 之间的关系可以用 Einstein 公式 ${ }^{[2]}$ 表示:

$$
\sigma=n e \mu=n e^{2} D /(k T)
$$

其中 $n$ 是单位体积的迁移电荷数; $e$ 是每个电子的 电量; $k$ 是玻尔兹曼常数.

假设带电载体穿过液滴的表面活性剂界面膜进 行扩散, 其活化能 $\left(E_{\mathrm{a}}\right)$ 可由Arrhenius -type 公式 ${ }^{200}$ 计算:

$$
D=\frac{4 l^{2} k T}{h} \exp \left(-\frac{E_{\mathrm{a}}}{R T}\right)
$$

其中 $l$ 是表面活性剂单分子层的厚度; $h$ 是普朗克 常数; $R$ 是气体常数. 由(2)和(3)式可推导出:

$$
\sigma=A \exp \left(-\frac{E_{\mathrm{a}}}{R T}\right)
$$

图 7 不同 $x$ 时温度对水/AOT/醇体系 $\ln \sigma$ 的影响

Fig.7 Influence of temperature on $\ln \sigma$ in the water/AOT/alkanols systems at different $x$ 
表 3 根据 Arrhenius-type 公式由 $\ln \sigma$ 对 1/T 作图计算的水/AOT/庚醇和水/AOT/癸醇体系活化能

Table 3 Activation energy estimated from the Arrhenius-type equation by plotting $\ln \sigma v s 1 / T$ in water/AOT/heptanol and water/AOT/decanol systems

\begin{tabular}{|c|c|c|c|c|c|c|}
\hline \multirow{2}{*}{$x$} & \multicolumn{3}{|c|}{$E_{\mathrm{a}}^{\circledR} /\left(\mathrm{kJ} \cdot \mathrm{mol}^{-1}\right)$} & \multicolumn{3}{|c|}{$E_{\mathrm{a}}^{2} /\left(\mathrm{kJ} \cdot \mathrm{mol}^{-1}\right)$} \\
\hline & $\mathrm{H}_{2} \mathrm{O}$ & $0.1 \mathrm{~mol} \cdot \mathrm{L}^{-1} \mathrm{NaSal}$ & $0.1 \mathrm{~mol} \cdot \mathrm{L}^{-1} \mathrm{NaC}$ & $\mathrm{H}_{2} \mathrm{O}$ & $0.1 \mathrm{~mol} \cdot \mathrm{L}^{-1} \mathrm{NaSal}$ & $0.1 \mathrm{~mol} \cdot \mathrm{L}^{-1} \mathrm{NaC}$ \\
\hline 1 & 14.16 & & & 13.29 & & \\
\hline 2 & 15.35 & 15.10 & 16.65 & 14.32 & 14.64 & 13.33 \\
\hline 5 & 19.42 & 18.23 & 18.44 & 17.22 & 16.56 & 17.32 \\
\hline 10 & 20.78 & 20.84 & 21.07 & 20.68 & 20.21 & 20.40 \\
\hline
\end{tabular}

(1) in water/AOT/heptanol systems, (2)in water/AOT/ decanol systems; $x$ is the same as Fig.l.

其中 $A=4 n e^{2} l^{2} / h$, 将(4) 式取对数, 通过 $\ln \sigma$ 对 $1 / T$ 作 图求出 $E_{\mathrm{a}}$. 温度对水/AOT/庚醇和水/AOT/癸醇体系 的 $\ln \sigma$ 的影响见图 7 , 所求 $E_{\mathrm{a}}$ 见表 3 .

对于水/AOT/癸醇体系, $x=1 、 2$ 对应渗滤前, $x=$ $5 、 10$ 对应渗滤后. 水/AOT/庚醇和水/AOT/癸醇体系 $\ln \sigma$ 都随温度升高而增加, 并与温度呈很好的线性 关系, 观察不到温度诱导渗滤现象. 两个体系活化能 均随 $x$ 增加而增加, 且相差不大, 均在 13.29 21.07 $\mathrm{kJ} \cdot \mathrm{mol}^{-1}$ 范围内, 与水/AOT/甲苯体系活化能 (13.9 $27.4 \mathrm{~kJ} \cdot \mathrm{mol}^{-1}$ )相近, 而大大低于水/AOT/烷烃活化能 (大于 $\left.100 \mathrm{~kJ} \cdot \mathrm{mol}^{-1}\right)^{[27]}$. 添加剂对活化能也基本没有影 响. 这表明在所研究的水量范围内体系的渗滤阀值 没有达到, 大量的 $\mathrm{Na}^{+}$迁移也只需越过很低的能垒, 因此没有观察到温度诱导的渗滤现象.

\section{3 结 论}

己醇、庚醇、辛醇和癸醇随其碳链增加分子体积 增加, 极性逐渐降低, 醇渗透进人胶束单分子层变得 困难, 醇的羟基与 AOT 极性头的相互作用力降低, 液滴间吸引力也相应变弱, 同时癸醇体系的黏度比 较大, 导致液滴扩散运动变慢, 因此只在水/AOT/癸 醇体系观察到渗滤现象. 加人胆酸钠使表面活性剂 界面排列得更加紧密, 限制了 $\mathrm{Na}^{+}$的迁移, 使电导率 降低; 水杨酸钠使表面活性剂界面膜柔性增加同时 增加了液滴中 $\mathrm{Na}^{+}$的浓度, 使电导率增加. 水/AOT/ 醇体系的活化能在 $13.29 \sim 21.07 \mathrm{~kJ} \cdot \mathrm{mol}^{-1}$, 大大低于 水/AOT/烷烃体系活化能, $\mathrm{Na}^{+}$迁移运动只需越过很 低的能垒, 因此没有观察到温度诱导渗滤现象.

\section{References}

1 Liu, D. J.; Ma, J. M.; Cheng, H. M.; Zhao, Z. G. Colloids Surfaces A, 1998, 143: 59

2 Li, Q.; Li, T.; Wu, J. G. Colloids Surfaces A, 2002, 197: 101

3 Jada., A.; Lang, J.; Zana, R. J. Phys. Chem., 1989, 93: 10

4 Bordi, F.; Cametti, C. Colloid. Polym Sci., 1998, 276: 1044
5 Liu, D. J.; Ma, J. M.; Cheng, H. M.; Zhao, Z. G. J. Disp. Sci. Technol., 1999, 20: 513

6 Ray, S.; Moulik, S. P. J. Colloid Interface Sci., 1995, 173: 28

7 Gradiielski, M.; Hoffmann, H.; Panitz, J. C.; Wokaun, A. J. Colloid Interface Sci., 1995, 169: 103

8 Zhang, X. G.; Dong, J. F.; Zhang, G. Y.; Hong, X. L.; Li, X. F. J. Colloid Interface Sci., 2005, 285: 336

9 Eicke, H. F.; Brokovic, M.; Dasgapta, B. J. Phys. Chem., 1989, 93: 314

10 Jada, A.; Lang, J.; Zana, R.; Makhlouffu, R.; Hirsch, E.; Candau, S. O. J. Phys. Chem., 1990, 94: 87

11 Moulik, S. P.; Pal, B. K. Adv. Colloid Interface Sci., 1998, 78: 99

12 Moulik, S. P.; De, G. C.; Bhowmik, B. B,; Panda, A. K. J. Phys. Chem. B, 1999, 103: 7122

13 Lagues, M.; Santerey, C. J. Phys. Chem., 1980, 84: 3503

14 Hilfilker, R.; Eicker, H. F.; Geiger, S.; Furlur, G. J. Colloid Interface Sci., 1985, 105: 378

15 Maitra, A. N.; Mathew, C.; Varshney, M. J. Phys. Chem., 1990, 94: 5290

16 Jada., A.; Lang, J.; Zana, R. J. Phys. Chem., 1990, 94: 387

17 Kataoka, H.; Eguchi, T.; Masui, H.; Miyakubo, K.; Nakayama, H.; Nakamura, N. J. Phys. Chem. B, 2003, 107: 12542

18 Mukhopadhyay, L.; Bhattcharya, P. K.; Moulik, S. P. Colloids Surfaces, 1990, 50: 295

19 Ray, S.; Bisal, S. R.; Moulik, S. P. J. Chem. Soc. Faraday Trans., 1993, 89: 3277

20 Ray, S.; Paul, S.; Moulik, S. P. J. Colloid Interface Sci., 1996, 183: 6

21 Nazário, L. M. M.; Hatton, T. A.; Crespo, J. P. S. G. Langmuir, 1996, $12: 6326$

22 Dutkiewicz, E.; Robinson, B. H. J. Electroanal. Chem., 1988, 251: 11

23 Ekwall, P.; Mandell, L.; Fontell, K. J. Colloid Interface Sci., 1970, 33: 215

24 Roy, B. K.; Moulik, S. P. Colloids Surfaces A, 2002, 203: 155

25 Fu, X.; Pan, Y.; Hu , Z. S.; Ma, Z. F. Colloids Surfaces A, 1996, 110: 55

26 Mathew, C.; Patanjali, P. K.; Nabi, A.; Maitra, A. Colloids Surfaces, 1988, 30: 253

27 Velazquez, M. M.; Valero, M.; Ortega, F. J. Phys. Chem. B, 2001, 105: 10163 